\title{
RENORMALIZATION OF THE LOCAL TIME FOR THE $d$-DIMENSIONAL FRACTIONAL BROWNIAN MOTION WITH $N$ PARAMETERS
}

\author{
M. EDDAHBI ${ }^{1}$, R. LACAYO, J. L. SOLÉ 2 , J. VIVES ${ }^{2}$ AND \\ C. A. TUDOR
}

\begin{abstract}
We study the asymptotic behavior in Sobolev norm of the local time of the $d$-dimensional fractional Brownian motion with $N$-parameters when the space variable tends to zero, both for the fixed time case and when simultaneously time tends to infinity and space variable to zero.
\end{abstract}

\section{$\S 1$. Introduction}

Let $B^{H}=\left\{B_{t}^{H}: t \geq 0\right\}$ be a standard fractional Brownian motion (fBm for brevity) with Hurst parameter $H \in(0,1)$. It is well known that this process is a centered Gaussian process which admits an integral representation of the form

$$
B_{t}^{H}=\int_{0}^{t} K_{H}(t, s) d W_{s}
$$

where $W$ is a standard Wiener process. The kernel $K_{H}(t, s)$ is given, for $s<t$, by

$$
K_{H}(t, s)=c_{H}(t-s)^{\mu}-\mu c_{H} \int_{s}^{t}(r-s)^{\mu-1}\left(1-\left(\frac{s}{r}\right)^{-\mu}\right) d r
$$

with $c_{H}$ being a constant and $\mu=H-\frac{1}{2}$.

The covariance function of $B_{t}^{H}$ can be represented as

$$
R_{H}(s, t)=\mathbb{E}\left(B_{s}^{H} B_{t}^{H}\right)=\int_{0}^{s \wedge t} K_{H}(t, r) K_{H}(s, r) d r
$$

Received June 10, 2005.

Revised January 25, 2006.

1991 Mathematics Subject Classification: 60H05, 60H10.

${ }^{1}$ Supported by the Spanish Ministerio de Educación, Cultura y Deporte: Grant SB2000-0060.

${ }^{2}$ Supported by the Spanish Ministerio de Educación, Cultura y Deporte: Grant BFM2000-0009 and by the Catalan CIRIT: Grant 2001SGR00174. 
and has the explicit form

$$
R_{H}(s, t)=\frac{1}{2}\left(s^{2 H}+t^{2 H}-|t-s|^{2 H}\right) .
$$

A very good survey about the $\mathrm{fBm}$ is the paper of Nualart [5].

For $\bar{H}=\left(H_{1}, \ldots, H_{N}\right)$ the $(N, 1)$-fBm is defined as

$$
B_{t}^{\bar{H}}=\int_{[0, t]} K_{\bar{H}}(t, s) d W_{s},
$$

where $K_{\bar{H}}(t, s)=\bigotimes_{j=1}^{N} K_{H_{j}}\left(t_{j}, s_{j}\right), s, t \in \mathbb{R}_{+}^{N}$ and $W$ is a standard $N$ parameter Brownian motion. Its covariance function is

$$
R_{\bar{H}}(s, t)=\mathbb{E}\left(B_{s}^{\bar{H}} B_{t}^{\bar{H}}\right)=\prod_{j=1}^{N} R_{H_{j}}\left(s_{j}, t_{j}\right) .
$$

Finally given the $N \times d$-matrix $\bar{H}=\left(\bar{H}_{1}, \ldots, \bar{H}_{d}\right)$ where for $i=1, \ldots, d$ and $j=1, \ldots, N, \bar{H}_{i}=\left(H_{i, 1}, \ldots, H_{i, N}\right)$ is a column vector and $H_{i, j} \in(0,1)$, the $N$-parameter, $d$-dimensional fractional Brownian motion $((N, d)$-fBm for brevity) is defined by $B^{\bar{H}}=\left(B_{t}^{\bar{H}_{1}}, \ldots, B_{t}^{\bar{H}_{d}}\right)_{t \in \mathbb{R}_{+}^{N}}$ where its components are independent and for every $i=1, \ldots, d, B^{\bar{H}_{i}}$ is a $(N, 1)$-fBm with Hurst parameter $\bar{H}_{i}$.

For any $t \in \mathbb{R}_{+}^{N}$ and $x \in \mathbb{R}^{d}$, the local time $L(t, x)$ of the $(N, d)$-fBm can be defined as the density of the occupation measure $\mu_{t}$, defined as

$$
\mu_{t}(A)=\int_{[0, t]} \mathbb{1}_{A}\left(B_{s}^{\bar{H}}\right) d s, \quad A \in \mathcal{B}\left(\mathbb{R}^{d}\right) .
$$

Formally, we can write

$$
L(t, x)=\int_{[0, t]} \delta_{x}\left(B_{s}^{\bar{H}}\right) d s
$$

where $\delta_{x}$ denotes the Dirac function and $\delta_{x}\left(B_{s}^{\bar{H}}\right)$ is therefore a distribution in the Watanabe sense (see [6]).

This local time for $(N, d)$-fBm has been studied by Xiao and Zhang [7], $\mathrm{Hu}$ and Oksendal [2] and Eddahbi et al. [1] between others.

The aim of this paper is to study the asymptotic behavior of $L(t, x)$ when $|x|$, the euclidean norm of $x$ in $\mathbb{R}^{d}$, goes to 0 , both for a fixed time and 
when the time goes to infinity, and we renormalize his Sobolev norm. We generalize the results of [3] from the $(N, d)$-standard Brownian motion to the $(N, d)$-fractional Brownian motion. In the standard Brownian motion case, the covariance function is simply $R_{\frac{1}{2}}(s, t)=s \wedge t$. Here, the control of the covariance function $R_{H}(s, t)$ for $H \neq \frac{1}{2}$ is the main difficulty.

Section 2 is devoted to the presentation of the problem. In particular we review from [1] the chaotic decomposition of the local time $L(t, x)$ as a functional of the $(N, d)-\mathrm{fBm}$ and its regularity in terms of SobolevWatanabe norms. In Section 3 we present a list of auxiliary lemmas. Section 4 is devoted to the presentation and proof of the main result, namely the asymptotic behavior of this local time, for fixed $t$, in the case $H_{i, j}=H$, $\forall i, j$, when $|x|$ goes to 0 . In Section 5 we extend the result to the case $\underline{t}:=t_{1} \cdots t_{N}$ going to infinity.

\section{$\S 2$. Preliminaries and statement of the problem}

If $F$ is a square integrable Brownian random variable, it can be represented by its Wiener chaos expansion

$$
F=\sum_{n=0}^{\infty} I_{n}\left(f_{n}\right)
$$

where $I_{n}\left(f_{n}\right)$ denotes the multiple Itô stochastic integral of the symmetric kernel $f_{n} \in L^{2}\left(\mathbb{R}_{+}^{n}\right)$ with respect to the Wiener process $W$.

If $\mathbf{L}$ is the Ornstein-Uhlenbeck operator

$$
\mathbf{L} F=-\sum_{n=0}^{\infty} n I_{n}\left(f_{n}\right)
$$

$p \in(1, \infty)$ and $\alpha \in \mathbb{R}$, we define the Sobolev-Watanabe spaces $\mathbb{D}^{\alpha, p}$ as the closure of the set of polynomial random variables with respect to the norm

$$
\|F\|_{\alpha, p}=\left\|(\mathbf{I d}-\mathbf{L})^{\frac{\alpha}{2}} F\right\|_{L^{p}(\Omega)},
$$

where Id stands for the identity mapping.

We denote by $D$ the chaotic derivative operator. It acts on multiple Itô stochastic integrals as

$$
D_{t}\left(I_{n}\left(f_{n}\right)\right)=n I_{n-1}\left(f_{n}(\cdot, t)\right)
$$

and is continuous from $\mathbb{D}^{\alpha, p}$ into $\mathbb{D}^{\alpha-1, p}\left(L^{2}\left(\mathbb{R}_{+}\right)\right)$. 
It is known that a Brownian random variable $F$ belongs to $\mathbb{D}^{\alpha, 2}$ if and only if its chaotic decomposition $\sum_{n=0}^{\infty} I_{n}\left(f_{n}\right)$ satisfies

$$
\sum_{n=0}^{\infty}(1+n)^{\alpha}\left\|I_{n}\left(f_{n}\right)\right\|_{2}^{2}<\infty
$$

where $\left\|I_{n}\left(f_{n}\right)\right\|_{2}^{2}=n !\left\|f_{n}\right\|_{2}^{2}$.

Set $\mathbb{D}^{\infty, 2}=\bigcap_{\alpha \in \mathbb{R}} \mathbb{D}^{\alpha, 2}$. If $F \in \mathbb{D}^{\infty, 2}$, we can compute its chaos expansion using the Stroock formula

$$
F=\sum_{n=0}^{\infty} \frac{1}{n !} I_{n}\left(\mathbb{E}\left(D^{n} F\right)\right) .
$$

For a complete survey of this subjects we refer the reader to the book of Watanabe [6].

Let $p_{\varepsilon}(x)$ be the centered Gaussian kernel with variance $\varepsilon>0$. Consider also, for $x \in \mathbb{R}^{d}$ and $\varepsilon>0$, the Gaussian kernel on $\mathbb{R}^{d}$ given by

$$
p_{\varepsilon}^{d}(x)=\prod_{i=1}^{d} p_{\varepsilon}\left(x_{i}\right), \quad x=\left(x_{1}, \ldots, x_{d}\right) .
$$

We denote by $\mathbf{H}_{n}$ the $n$-th Hermite polynomial, defined for $n \geq 1$, by

$$
\mathbf{H}_{n}(x)=\frac{(-1)^{n}}{n !} \exp \left(\frac{x^{2}}{2}\right) \frac{d^{n}}{d x^{n}}\left(\exp \left(-\frac{x^{2}}{2}\right)\right), \quad x \in \mathbb{R}
$$

and $\mathbf{H}_{0}(x)=1$.

As we proved in [1] the chaotic decomposition of the local time of the $(N, d)-\mathrm{fBm}$ is

$$
L(t, x)=\sum_{n_{1}, \ldots, n_{d} \geq 0} \int_{[0, t]} \prod_{i=1}^{d} \frac{p^{2}{ }^{2 \bar{H}_{i}}\left(x_{i}\right)}{\underline{s}^{n_{i} \bar{H}_{i}}} \mathbf{H}_{n_{i}}\left(\frac{x_{i}}{\underline{s}^{\bar{H}_{i}}}\right) I_{n_{i}}^{i}\left(K_{\bar{H}_{i}}(s, \cdot)^{\otimes n_{i}}\right) d s,
$$

provided that $\sum_{j=1}^{N} \frac{1}{H_{j}^{*}}>d$, where $t \in \mathbb{R}_{+}^{N}, x \in \mathbb{R}^{d} \backslash\{0\}, \underline{s}=s_{1} \cdots s_{N}$, $\underline{s}^{\bar{H}_{i}}=\prod_{j=1}^{N} s_{j}^{H_{i, j}}$ and $H_{j}^{*}=\max \left\{H_{i, j}, i=1, \ldots, d\right\}$. The integrals $I_{n_{i}}^{i}$ denotes the multiple Itô stochastic integrals with respect to the independent $N$-parameter Wiener processes $W^{i}$.

Moreover, in [1] we proved that this functional belongs to the space $\mathbb{D}^{\alpha, 2}$ if

$$
\alpha<\sum_{j=1}^{N} \frac{1}{2 H_{j}^{*}}-\frac{d}{2}
$$


If all $H_{i, j}=H$, this expression becomes $\alpha<\frac{N}{2 H}-\frac{d}{2}$, and then a sufficient condition for the local time to be in $L^{2}(\Omega)$ is $N>H d$. Observe that this sufficient condition is also founded in Xiao and Zhang [7]. From now on we will suppose always this condition.

Recall that if $H=\frac{1}{2}, \sum_{j=1}^{N} \frac{1}{2 H_{j}^{*}}-\frac{d}{2}=N-\frac{d}{2}$, which is the same condition obtained in [3] for the $N$-parameter Wiener process in $\mathbb{R}^{d}$.

\section{§3. Auxiliary lemmas}

LEMMA 1. If $\frac{1}{4} \leq \beta \leq \frac{1}{2}$ we have

$$
\sup _{x \in \mathbb{R}}\left|\sqrt{n !} \mathbf{H}_{n}(x) e^{-\beta x^{2}}\right| \leq c(n \vee 1)^{-\frac{8 \beta-1}{12}} .
$$

Proof. This result is proved in [4].

Remark 2. The factor $\sqrt{n !}$ appears because we do not use the same definition of Hermite polynomials as in [4].

Lemma 3. Let $d \geq 1$ and $\nu \in(0,1)$. We can choose a universal constant $c$ such that for any $m \geq 1$,

$$
\sum_{n_{1}+\cdots+n_{d}=m} \prod_{i=1}^{d}\left(n_{i} \vee 1\right)^{-\nu} \leq c m^{d(1-\nu)-1} .
$$

Proof. This result is proved in [4].

Lemma 4. Let $\gamma$ and $a$ be positive constants and $b \in \mathbb{R}$. Set $\alpha=\frac{b-1}{a}$. Then

$$
\int_{[0,1]^{N}} \exp \left(-\frac{\gamma}{\underline{s}^{a}}\right) \frac{d s}{\underline{s}^{b}}=\frac{1}{(N-1) !}\left(\frac{1}{a}\right)^{N} \gamma^{-\alpha} g_{N-1}(\gamma, \alpha)
$$

where

$$
g_{N-1}(\gamma, \alpha):=\int_{\gamma}^{\infty} t^{\alpha-1} e^{-t}\left(\log \frac{t}{\gamma}\right)^{N-1} d t .
$$

Proof. Using the change of variables $u_{1}=s_{1} \cdots s_{N}, u_{2}=s_{2} \cdots s_{N}, \ldots$, $u_{N}=s_{N}$, with Jacobi determinant $\frac{1}{u_{2} \cdots u_{N}}$, we obtain

$$
\begin{aligned}
\int_{[0,1]^{N}} \exp \left(-\frac{\gamma}{\underline{s}^{a}}\right) \frac{d s}{\underline{s}^{b}} & =\int_{\left\{0 \leq u_{1} \leq \cdots \leq u_{N} \leq 1\right\}} \frac{1}{u_{1}^{b}} \exp \left(-\frac{\gamma}{u_{1}^{a}}\right) \frac{d u_{N} \cdots d u_{2}}{u_{N} \cdots u_{2}} d u_{1} \\
& =\frac{1}{(N-1) !} \int_{0}^{1}\left(\log \frac{1}{r}\right)^{N-1} \frac{1}{r^{b}} \exp \left(-\frac{\gamma}{r^{a}}\right) d r,
\end{aligned}
$$

and making the change of variable $\gamma r^{-a}=t$ we get the desired result. 
Lemma 5. The function

$$
Q_{H}(z)= \begin{cases}\frac{R_{H}(1, z)}{z^{H}} & \text { if } z \in(0,1] \\ 0 & \text { if } z=0,\end{cases}
$$

has the following properties:

1. It is strictly increasing and it continuously maps $[0,1]$ onto $[0,1]$. Moreover, $Q_{H}(1)=1$.

2. For fixed $\delta \in(0,1)$ and for any $z \in[0,1-\delta]$, it satisfies the inequality

$$
Q_{H}(z) \leq c(H, \delta) z^{G}
$$

where $G=H \wedge(1-H)$.

3. For fixed $\delta \in(0,1)$ and $\beta>0$, it satisfies the inequality

$$
\int_{1-\delta}^{1} Q_{H}(z)^{\beta} d z \leq \frac{c(H, \delta)}{\beta^{\frac{1}{2 H}}} .
$$

Proof. The proof of parts 1 and 3 are done in [1].

For the part 2 we have

$$
Q_{H}(z)=\frac{1-(1-z)^{2 H}}{2 z^{H}}+\frac{z^{H}}{2} .
$$

Using Taylor expansion, $1-(1-z)^{2 H}=2 H(1-\theta)^{2 H-1} z$ with $0 \leq \theta \leq z$.

If $H \geq \frac{1}{2}$, we have $1-(1-z)^{2 H} \leq 2 H z$, and therefore $Q_{H}(z) \leq$ $H z^{1-H}+\frac{1}{2} z^{H} \leq c_{1} z^{1-H}, c_{1}$ being a positive constant.

If $H<\frac{1}{2}$ and $z \in[0,1-\delta]$, we have $1-(1-z)^{2 H} \leq 2 H \delta^{2 H-1} z$, and then $Q_{H}(z) \leq H \delta^{2 H-1} z^{1-H}+\frac{1}{2} z^{H} \leq c_{2} z^{H}, c_{2}$ being another positive constant.

In what follows, for every $x>0$ and $\gamma \geq 0$, we denote the complementary incomplete Gamma function as

$$
\Gamma(x, \gamma)=\int_{\gamma}^{\infty} e^{-t} t^{x-1} d t
$$

In particular $\Gamma(x):=\Gamma(x, 0)$ and $\Gamma(x, \gamma) \leq \Gamma(x)$. 
LEMMA 6. The function

$$
g_{N-1}(\gamma, \alpha):=\int_{\gamma}^{\infty} t^{\alpha-1} e^{-t}\left(\log \frac{t}{\gamma}\right)^{N-1} d t, \quad N \in \mathbb{N}
$$

has the following behavior when $\gamma$ tends to 0 :

1. If $\alpha>0$ and $N \geq 2, g_{N-1}(\gamma, \alpha)=\left(\log \frac{1}{\gamma}\right)^{N-1} \Gamma(\gamma, \alpha)+\mathcal{O}\left(\left(\log \frac{1}{\gamma}\right)^{N-2}\right)$. If $N=1$, it tends to $\Gamma(\alpha)$

2. If $\alpha=0$ and $N \geq 2, g_{N-1}(\gamma, \alpha)=e^{-\gamma} \frac{1}{N}\left(\log \frac{1}{\gamma}\right)^{N}+\mathcal{O}\left(\left(\log \frac{1}{\gamma}\right)^{N-1}\right)$. If $N=1$, it behaves as $e^{-\gamma} \ln \frac{1}{\gamma}$

3. If $\alpha<0$ and $N \geq 2, g_{N-1}(\gamma, \alpha)=\gamma^{\alpha}\left(\frac{\Gamma(N)}{|\alpha|^{N}}+o(\gamma)\right)$. If $N=1$, its behavior is as $\frac{1}{|\alpha|} \gamma^{\alpha}$

Proof. The cases with $N=1$ are straightforward. For $N \geq 2$, note that

$$
\left(\log \frac{t}{\gamma}\right)^{N-1}=\sum_{k=0}^{N-1}\left(\begin{array}{c}
N-1 \\
k
\end{array}\right)\left(\log \frac{1}{\gamma}\right)^{N-1-k}(\log t)^{k}
$$

Then,

- If $\alpha>0$, the function

$$
t \longmapsto t^{\frac{\alpha}{2}-1} e^{-t}(\log t)^{k}
$$

is always integrable on $[0, \infty)$ for any $k \in \mathbb{N} \cup\{0\}$. Therefore,

$$
g_{N-1}(\gamma, \alpha)=\left(\log \frac{1}{\gamma}\right)^{N-1} \Gamma(\gamma, \alpha)+\mathcal{O}\left(\left(\log \frac{1}{\gamma}\right)^{N-2}\right)
$$

- If $\alpha=0$, we need to estimate the integral

$$
g_{N-1}(\gamma, 0)=\int_{\gamma}^{\infty} t^{-1} e^{-t}\left(\log \frac{t}{\gamma}\right)^{N-1} d t
$$

Integrating by parts we obtain

$$
\begin{aligned}
g_{N-1}(\gamma, 0) & =\frac{1}{N} \int_{\gamma}^{\infty} e^{-t}\left(\log \frac{t}{\gamma}\right)^{N} d t \\
& =\frac{e^{-\gamma}}{N}\left(\log \frac{1}{\gamma}\right)^{N}+\mathcal{O}\left(\left(\log \frac{1}{\gamma}\right)^{N-1}\right) .
\end{aligned}
$$

- If $\alpha<0$, making the change of variable $s=-\alpha \log \left(\frac{t}{\gamma}\right)$, the result follows immediately. 


\section{§4. Renormalization of the local time for fixed $t$}

The main purpose of this section is to study the asymptotic behavior of $L(t, x)$, for $t \in \mathbb{R}_{+}^{N}$ and $x \in \mathbb{R}^{d} \backslash\{0\}$, when $|x| \rightarrow 0$. In the case $d H \geq 1$ it has a singularity. An interesting question is to renormalize the local time, that means, to find a deterministic function $f(t, x)$ such that $f(t, x) L(t, x)$ converge to 1 in some precise sense. We will do it with respect the norm $\|\cdot\|_{\alpha, 2}$. Then we will obtain a function $f(t, x)$ such that $\|f(t, x) L(t, x)\|_{\alpha, 2}$ converges to 1 when $|x| \rightarrow 0$, both for fixed $t$ and when $\underline{t}=t_{1} \cdots t_{N} \rightarrow \infty$.

Recall the expression of the $\mathbb{D}^{\alpha, 2}$-norm of the local time $L(t, x)$. For the sake of simplicity we take $t=\widetilde{1}:=(1, \ldots, 1)$.

We have

$$
\|L(\widetilde{1}, x)\|_{\alpha, 2}^{2}=\sum_{m=0}^{\infty}(1+m)^{\alpha} A_{m}(x)
$$

where

$$
\begin{aligned}
A_{m}(x)= & \sum_{n_{1}+\cdots+n_{d}=m} \| \int_{[0,1]^{N}} \prod_{i=1}^{d} \frac{\underline{p}^{2 \bar{H}_{i}}\left(x_{i}\right)}{\underline{s}^{n_{i} \bar{H}_{i}}} \\
& \times \mathbf{H}_{n_{i}}\left(\frac{x_{i}}{\underline{s}^{\bar{H}_{i}}}\right) I_{n_{i}}^{i}\left(K_{\bar{H}_{i}}(s, \cdot)^{\otimes n_{i}}\right) d s \|_{L^{2}(\Omega)}^{2},
\end{aligned}
$$

and as

$$
\begin{gathered}
E\left(I_{n_{i}}^{i}\left(K_{\bar{H}_{i}}(u, \cdot)^{\otimes n_{i}}\right) I_{n_{j}}^{j}\left(K_{\bar{H}_{j}}(v, \cdot)^{\otimes n_{j}}\right)\right)=\delta_{i j} n_{i} !\left(R_{\bar{H}_{i}}(u, v)\right)^{n_{i}} \\
A_{m}(x)=\sum_{n_{1}+\cdots+n_{d}=m} \int_{[0,1]^{N}} d u \int_{[0,1]^{N}} d v \prod_{i=1}^{d}\left(\prod_{j=1}^{N} \frac{R_{H_{i, j}}\left(u_{j}, v_{j}\right)}{\left(u_{j} v_{j}\right)^{H_{i, j}}}\right)^{n_{i}} \\
\times n_{i} ! \mathbf{H}_{n_{i}}\left(\frac{x_{i}}{\underline{u}^{\bar{H}_{i}}}\right) \mathbf{H}_{n_{i}}\left(\frac{x_{i}}{\underline{v}^{\bar{H}_{i}}}\right) p_{\underline{u}^{2} \bar{H}_{i}}\left(x_{i}\right) p_{\underline{v}^{2} \bar{H}_{i}}\left(x_{i}\right),
\end{gathered}
$$

and in particular

$$
A_{0}(x)=\left(\int_{[0,1]^{N}} d s \prod_{i=1}^{d} \frac{1}{\left(2 \pi \prod_{j=1}^{N} s_{j}^{2 H_{i, j}}\right)^{\frac{1}{2}}} \exp \left(-\frac{x_{i}^{2}}{2 \prod_{j=1}^{N} s_{j}^{2 H_{i, j}}}\right)\right)^{2} .
$$

In all this section we confine our attention to the situation where $H_{i, j}=$ $H$ for all $(i, j) \in\{1, \ldots, d\} \times\{1, \ldots, N\}$, and use the notation $B^{H}$ for $B^{\bar{H}}$. 
Observe that in this particular case

$$
A_{0}(x)=\frac{1}{(2 \pi)^{d}}\left(\int_{[0,1]^{N}} \frac{1}{\underline{s}^{d H}} \exp \left(-\frac{|x|^{2}}{2 \underline{s}^{2 H}}\right) d s\right)^{2},
$$

and

$$
\begin{aligned}
A_{m}(x)= & \sum_{n_{1}+\cdots+n_{d}=m} \int_{[0,1]^{N}} \int_{[0,1]^{N}}\left(\prod_{j=1}^{N} \frac{R_{H}\left(u_{j}, v_{j}\right)}{\left(u_{j} v_{j}\right)^{H}}\right)^{m} \\
& \times \prod_{i=1}^{d} n_{i} ! \mathbf{H}_{n_{i}}\left(\frac{x_{i}}{\underline{u}^{H}}\right) \mathbf{H}_{n_{i}}\left(\frac{x_{i}}{\underline{v}^{H}}\right) p_{\underline{u}^{2 H}}\left(x_{i}\right) p_{\underline{v}^{2 H}}\left(x_{i}\right) d u d v
\end{aligned}
$$

Our main result is the following:

Theorem 7. Let $B^{H}$ be $(N, d)-f B m$. Set $\lambda:=d-\frac{1}{H}$. For any $\alpha<$ $\frac{N}{2 H}-\frac{d}{2}$ we have:

1) If $\lambda>0$ and $N \geq 2$,

$$
\lim _{|x| \rightarrow 0}\|L(\widetilde{1}, x)\|_{\alpha, 2}\left(\frac{2^{\frac{\lambda}{2}}\left(\frac{1}{2 H}\right)^{N}|x|^{-\lambda}}{(2 \pi)^{\frac{d}{2}}(N-1) !}\left(\log \frac{2}{|x|^{2}}\right)^{N-1} \Gamma\left(\frac{\lambda}{2}\right)\right)^{-1}=1 .
$$

If $N=1,\|L(1, x)\|_{\alpha, 2}$ explodes when $x$ tends to 0 , and $\|L(1, x)\|_{\alpha, 2}$. $\left(|x|^{-\lambda}\right)^{-1}$ is bounded by a positive constant.

2) If $\lambda=0$,

$$
\lim _{|x| \rightarrow 0}\|L(\widetilde{1}, x)\|_{\alpha, 2}\left(\frac{\left(\frac{1}{2 H}\right)^{N}}{(2 \pi)^{\frac{d}{2}} N !}\left(\log \frac{2}{|x|^{2}}\right)^{N}\right)^{-1}=1 .
$$

3) If $\lambda<0$,

$$
\begin{aligned}
& \lim _{|x| \rightarrow 0}\|L(\widetilde{1}, x)\|_{\alpha, 2}=\|L(\widetilde{1}, 0)\|_{\alpha, 2}=\frac{1}{(2 \pi)^{\frac{d}{2}}}\left(\frac{1}{1-H d}\right)^{\frac{N}{2}} \\
& \times\left[\sum_{r=0}^{\infty}(1+2 r)^{\alpha} \sum_{r_{1}+\cdots+r_{d}=r} \prod_{i=1}^{d} \frac{\left(2 r_{i}\right) !}{\left(r_{i} !\right)^{2} 2^{2 r_{i}}}\left(\int_{0}^{1} Q_{H}(z)^{m} \frac{d z}{z^{d H}}\right)^{N}\right]^{\frac{1}{2}}<\infty .
\end{aligned}
$$

Remark 8. This theorem shows that for $\lambda \geq 0$ the local time explodes at the origin and for $\lambda<0$ it does not. Observe that for the $N$-Brownian process, that is $H=\frac{1}{2}$, the local time explodes at the origin if and only if $d \geq 2$, and we obtain, with another proof, the same results as Imkeller and Weisz [3]. 
Proof. The idea of the proof is to show that the convergence of $A_{m}(x)$ for any $m \geq 1$ when $|x| \rightarrow 0$, is controlled by $A_{0}(x)$ and then the asymptotic behavior of $L(1, x)$ coincides with the asymptotic behavior of $A_{0}(x)^{\frac{1}{2}}$.

Define now, for $\gamma>0$ and $m \geq 0$,

$$
B_{m}(\gamma)=\int_{[0,1]^{N}} \int_{[0,1]^{N}} \frac{\left(\prod_{j=1}^{N} R_{H}\left(u_{j}, v_{j}\right)\right)^{m}}{(\underline{u} \cdot \underline{v})^{H(m+d)}} \exp \left(-\frac{\gamma}{\underline{u}^{2 H}}\right) \exp \left(-\frac{\gamma}{\underline{v}^{2 H}}\right) d u d v
$$

Clearly,

$$
A_{0}(x)=\frac{1}{(2 \pi)^{d}} B_{0}\left(\frac{1}{2}|x|^{2}\right)
$$

For $m \geq 1$, choosing $\beta \in\left[\frac{1}{4}, \frac{1}{2}\right)$, we can write

$$
\begin{aligned}
A_{m}(x)= & \frac{1}{(2 \pi)^{d}} \sum_{n_{1}+\cdots+n_{d}=m} \int_{[0,1]^{N}} \int_{[0,1]^{N}}\left(\prod_{j=1}^{N} \frac{R_{H}\left(u_{j}, v_{j}\right)}{\left(u_{j} v_{j}\right)^{H}}\right)^{m} \frac{1}{(\underline{u v})^{d H}} \\
& \times \prod_{i=1}^{d} \sqrt{n_{i} !} \mathbf{H}_{n_{i}}\left(\frac{x_{i}}{\underline{u}^{H}}\right) \exp \left\{-\beta \frac{x_{i}^{2}}{\underline{u}^{2 H}}\right\} \sqrt{n_{i} !} \mathbf{H}_{n_{i}}\left(\frac{x_{i}}{\underline{v}^{H}}\right) \exp \left\{-\beta \frac{x_{i}^{2}}{\underline{v}^{2 H}}\right\} \\
& \times \exp \left\{-\left(\frac{1}{2}-\beta\right) \frac{x_{i}^{2}}{\underline{u}^{2 H}}\right\} \exp \left\{-\left(\frac{1}{2}-\beta\right) \frac{x_{i}^{2}}{\underline{v}^{2 H}}\right\} d u d v,
\end{aligned}
$$

and applying Lemmas 1 and 3 we obtain

$$
A_{m}(x) \leq c \frac{1}{(2 \pi)^{d}} m^{d\left(1-\frac{8 \beta-1}{6}\right)-1} B_{m}\left(\left(\frac{1}{2}-\beta\right)|x|^{2}\right) .
$$

Then our problem reduces to the study of the asymptotic behavior of $B_{m}$.

As $R_{H}\left(u_{j}, v_{j}\right)=R_{H}\left(1, \frac{v_{j}}{u_{j}}\right) u_{j}^{2 H}$, we have

$$
\begin{aligned}
B_{m}(\gamma)=2^{N} & \int_{[0,1]^{N}} \int_{0}^{u_{N}} \cdots \int_{0}^{u_{1}} \prod_{j=1}^{N} \frac{R_{H}\left(1, \frac{v_{j}}{u_{j}}\right)^{m} u_{j}^{2 H m}}{\left(u_{j} v_{j}\right)^{H(m+d)}} \\
& \times \exp \left(-\frac{\gamma}{\underline{u}^{2 H}}\right) \exp \left(-\frac{\gamma}{\underline{v}^{2 H}}\right) d v_{1} \cdots d v_{N} d u .
\end{aligned}
$$

With the change $\frac{v_{j}}{u_{j}}=z_{j},(j=1, \ldots, N)$ and computing iteratively the 
previous integral, we find

$$
\begin{aligned}
B_{m}(\gamma)=2^{N} & \int_{[0,1]^{N}}\left(\int_{[0,1]^{N}} \underline{u}^{1-2 H d} \exp \left(\frac{-\kappa(\underline{z}) \gamma}{\underline{u}^{2 H}}\right) d u_{1} \cdots d u_{N}\right) \\
& \times \prod_{j=1}^{N} \frac{R_{H}\left(1, z_{j}\right)^{m}}{z_{j} H(m+d)} d z_{1} \cdots d z_{N}
\end{aligned}
$$

where $\kappa(r)=1+\frac{1}{r^{2 H}}$.

By Lemma 4, with $a=2 H$ and $b=2 H d-1$, we have

$$
\begin{aligned}
J_{N}(\gamma, \underline{z}) & =\int_{[0,1]^{N}} \underline{u}^{1-2 H d} \exp \left(\frac{-\kappa(\underline{z}) \gamma}{\underline{u}^{2 H}}\right) d u \\
& =c(N, d, H) \gamma^{-\lambda} \int_{\gamma}^{\infty} e^{-s \kappa(\underline{z})} s^{\lambda-1}\left(\log \frac{s}{\gamma}\right)^{N-1} d s
\end{aligned}
$$

where $\lambda=d-\frac{1}{H}=\frac{b-1}{a}$.

Therefore

$$
\begin{aligned}
B_{m}(\gamma)=c(N, H, d) \gamma^{-\lambda} \int_{\gamma}^{\infty} \int_{[0,1]^{N}} \prod_{j=1}^{N} \frac{R_{H}\left(1, z_{j}\right)^{m}}{z_{j}^{H m}} \cdot \frac{e^{-\frac{s}{z^{2 H}}}}{\underline{z}^{H d}} \\
\times e^{-s} s^{\lambda-1}\left(\log \frac{s}{\gamma}\right)^{N-1} d z d s .
\end{aligned}
$$

First we will see that for $m>\frac{\lambda H}{G}$, we have

$$
B_{m}(\gamma) \leq c(H, d, N) \gamma^{-\lambda} g_{N-1}(\gamma, \lambda) m^{-\frac{N}{2 H}}
$$

Indeed, controlling the exponential by 1 , we obtain

$$
\begin{aligned}
B_{m}(\gamma) & \leq c(N, H, d) \gamma^{-\lambda} \int_{[0,1]^{N}} \prod_{j=1}^{N} \frac{R_{H}\left(1, z_{j}\right)^{m}}{z_{j}^{H(m+d)}} \int_{\gamma}^{\infty} e^{-s} s^{\lambda-1}\left(\log \frac{s}{\gamma}\right)^{N-1} d z d s \\
& =c(N, H, d) \gamma^{-\lambda} g_{N-1}(\gamma, \lambda)\left(\int_{0}^{1} Q_{H}(z)^{m} \frac{1}{z^{d H}} d z\right)^{N}
\end{aligned}
$$

where the function $Q_{H}$ is introduced in Lemma 5 .

Now, choosing $\delta \in(0,1)$, we have

$$
\int_{0}^{1} Q_{H}(z)^{m} \frac{1}{z^{d H}} d z \leq \int_{0}^{1-\delta} Q_{H}(z)^{m} \frac{1}{z^{d H}} d z+(1-\delta)^{-d H} \int_{1-\delta}^{1} Q_{H}(z)^{m} d z .
$$


The second summand on the right, using part 3 of Lemma 5 , is controlled by $c(H, \delta) m^{-\frac{1}{2 H}}$.

For the first summand, if $m>\frac{d H-1}{G}=\frac{\lambda H}{G}$, we fix $\alpha \in\left(\frac{\lambda H}{G}, m\right)$, and write

$$
\int_{0}^{1-\delta} Q_{H}(z)^{m} \frac{1}{z^{d H}} d z=\int_{0}^{1-\delta} Q_{H}(z)^{m-\alpha} Q_{H}(z)^{\alpha} \frac{1}{z^{d H}} d z .
$$

Using that $Q_{H}$ is an increasing function and part 2 of Lemma 5, we control this by

$$
Q_{H}(1-\delta)^{m-\alpha} c(H, \delta, \alpha) \int_{0}^{1-\delta} z^{\alpha G-d H} d z .
$$

As $\alpha>\frac{\lambda H}{G}$, the integral that appears in the last expression is a constant that depends on $H, d, \alpha$ and $\delta$.

Therefore, being $Q_{H}(1-\delta)<1$, we can estimate this term by

$$
c(H, d, \delta, \alpha) m^{-\frac{1}{2 H}},
$$

and we get (4).

Note that this result is true only for $m>\frac{\lambda H}{G}$. If $\lambda \leq 0$ this covers all cases. But if $\lambda>0$ the $B_{m}$ terms with $m \leq \frac{\lambda H}{G}$ are not controlled yet. The following part of the proof will discuss these first terms. From now on in each expression $c$ will be the suitable constant.

Observe that for any $0<\epsilon<m$, being $Q_{H}(\cdot) \leq 1$, we have

$$
B_{m}(\gamma) \leq B_{\epsilon}(\gamma)
$$

Now we will see that for $\lambda>0$,

$$
B_{\epsilon}(\gamma) \leq c(H, d, N) \gamma^{-\lambda}\left(g_{N-1}(\gamma, \lambda)+g_{N-1}(\gamma, \alpha)\right)
$$

where $\alpha$ is some positive constant depending also on $\epsilon$.

Indeed, putting $c=c(N, H, d)$,

$$
\begin{aligned}
B_{\epsilon}(\gamma)=c \gamma^{-\lambda} \int_{\gamma}^{\infty} \int_{[0,1]^{N}} \prod_{j=1}^{N} Q_{H}\left(z_{j}\right)^{\epsilon} \cdot \frac{e^{-\frac{s}{\underline{z}^{2 H}}}}{\underline{z}^{H d}} e^{-s} s^{\lambda-1}\left(\log \frac{s}{\gamma}\right)^{N-1} d z d s \\
=c \gamma^{-\lambda} \int_{\gamma}^{\infty} \sum_{k=0}^{N}\left(\begin{array}{c}
N \\
k
\end{array}\right) \underbrace{\int_{0}^{1-\delta} \cdots \int_{0}^{1-\delta}}_{k} \underbrace{\int_{1-\delta}^{1} \cdots \int_{1-\delta}^{1}}_{N-k} \prod_{j=1}^{N} Q_{H}\left(z_{j}\right)^{\epsilon} \cdot \frac{e^{-\frac{s}{z^{2 H}}}}{\underline{z}^{H d}} \\
\times e^{-s} s^{\lambda-1}\left(\log \frac{s}{\gamma}\right)^{N-1} d z_{1} \cdots d z_{k} d s,
\end{aligned}
$$


because the function

$$
\prod_{j=1}^{N} Q_{H}\left(z_{j}\right)^{\epsilon} \cdot \frac{e^{-\frac{s}{\underline{z}^{2 H}}}}{\underline{z}^{H d}},
$$

is symmetric in $z$.

Now estimating $Q_{H}$ and the exponential by 1 in the integrals between $1-\delta$ and 1 , we obtain

$$
\begin{aligned}
& B_{\epsilon}(\gamma) \leq c \gamma^{-\lambda} \int_{\gamma}^{\infty}\left(\left(\frac{\delta}{(1-\delta)^{d H}}\right)^{N}+\sum_{k=1}^{N}\left(\begin{array}{c}
N \\
k
\end{array}\right) \int_{0}^{1-\delta} \cdots \int_{0}^{1-\delta} \frac{\delta^{N-k}}{(1-\delta)^{d H(N-k)}}\right. \\
&\left.\times \prod_{j=1}^{k} Q_{H}\left(z_{j}\right)^{\epsilon} \cdot \frac{e^{-\frac{s}{\left(z_{1} \cdots z_{k}\right)^{2 H}}}}{\left(z_{1} \cdots z_{k}\right)^{H d}} d z_{1} \cdots d z_{k}\right) e^{-s} s^{\lambda-1}\left(\log \frac{s}{\gamma}\right)^{N-1} d s \\
& \leq c \gamma^{-\lambda} \int_{\gamma}^{\infty}\left(\left(\frac{\delta}{(1-\delta)^{d H}}\right)^{N}+\sum_{k=1}^{N}\left(\begin{array}{c}
N \\
k
\end{array}\right) \int_{0}^{1-\delta} \cdots \int_{0}^{1-\delta}\left(\frac{\delta}{(1-\delta)^{d H}}\right)^{N-k}\right. \\
&\left.\quad \times c(H, \delta)^{k \epsilon} \prod_{j=1}^{k} z_{j}^{\epsilon G-d H} e^{-\frac{s}{\left(z_{1} \cdots z_{k}\right)^{2 H}}} d z_{1} \cdots d z_{k}\right) e^{-s} s^{\lambda-1}\left(\log \frac{s}{\gamma}\right)^{N-1} d s
\end{aligned}
$$

where we have used part 2 of Lemma 5.

Now, choosing $\epsilon<\frac{d H}{G}$, we can use Lemma 4 with $a=2 H, b=-\epsilon G+$ $d H, \gamma=s, N=k$ and $\alpha=\frac{d H-\epsilon G-1}{2 H}$, to bound the right hand side of the last inequality by

$$
\begin{gathered}
c \gamma^{-\lambda}\left\{g_{N-1}(\gamma, \lambda)+\int_{\gamma}^{\infty} \sum_{k=1}^{N} \int_{s}^{\infty}\left(\log \frac{t}{s}\right)^{k-1} s^{\frac{\lambda}{2}+\frac{\epsilon G}{2 H}-1} t^{\frac{\lambda}{2}-\frac{\epsilon G}{2 H}-1}\right. \\
\left.\times e^{-t} e^{-s}\left(\log \frac{s}{\gamma}\right)^{N-1} d t d s\right\},
\end{gathered}
$$

where $c$ is a constant that depends on $H, d, N, \epsilon, \delta$.

Using the fact that for any $n \geq 1$ and for $t \geq s$ we have

$$
\log \frac{t}{s} \leq n\left(\frac{t}{s}\right)^{\frac{1}{n}}
$$

and taking $n=M(k-1)$ for a big $M$, we obtain

$$
\begin{aligned}
& B_{\epsilon}(\gamma) \leq c \gamma^{-\lambda}\left\{g_{N-1}(\gamma, \lambda)+\int_{\gamma}^{\infty} \int_{s}^{\infty} s^{\frac{\lambda}{2}+\frac{\epsilon G}{2 H}-1-\frac{1}{M}} t^{\frac{\lambda}{2}-\frac{\epsilon G}{2 H}-1+\frac{1}{M}}\right. \\
& \left.\times e^{-t} e^{-s}\left(\log \frac{s}{\gamma}\right)^{N-1} d t d s\right\}
\end{aligned}
$$


where now $c$ depends also on $M$.

As $\epsilon<m<\frac{\lambda H}{G}$, we have $\frac{\lambda}{2}-\frac{\epsilon G}{2 H}+\frac{1}{M}>0$ and

$$
\begin{aligned}
B_{\epsilon}(\gamma) \leq c \gamma^{-\lambda}\left\{g_{N-1}(\gamma, \lambda)+\int_{\gamma}^{\infty}\right. & s^{\frac{\lambda}{2}+\frac{\epsilon G}{2 H}-1-\frac{1}{M}} e^{-s} \Gamma\left(\frac{\lambda}{2}-\frac{\epsilon G}{2 H}+\frac{1}{M}, s\right) \\
& \left.\times\left(\log \frac{s}{\gamma}\right)^{N-1} d s\right\} .
\end{aligned}
$$

Controlling the complementary incomplete Gamma function by the corresponding Gamma function we obtain

$$
\begin{aligned}
B_{\epsilon}(\gamma) & \leq c \gamma^{-\lambda}\left\{g_{N-1}(\gamma, \lambda)+\int_{\gamma}^{\infty} s^{\frac{\lambda}{2}+\frac{\epsilon G}{2 H}-1-\frac{1}{M}} e^{-s}\left(\log \frac{s}{\gamma}\right)^{N-1} d s\right\} \\
& =c \gamma^{-\lambda}\left\{g_{N-1}(\gamma, \lambda)+g_{N-1}\left(\gamma, \frac{\lambda}{2}+\frac{\epsilon G}{2 H}-\frac{1}{M}\right)\right\} .
\end{aligned}
$$

Observe that for $M$ sufficiently large

$$
\frac{\lambda}{2}+\frac{\epsilon G}{2 H}-\frac{1}{M}>0
$$

Finally for the $m=0$ case, using Lemma 4 , we have immediately, as $\alpha=\frac{\lambda}{2}$

$$
B_{0}(\gamma)=\frac{1}{((N-1) !)^{2}} \frac{1}{(2 H)^{2 N}} \gamma^{-\lambda} g_{N-1}\left(\gamma, \frac{\lambda}{2}\right)^{2}
$$

Therefore we have to separate the cases $\lambda \geq 0$ and $\lambda<0$.

For $\lambda \geq 0$ we have

$$
\|L(\widetilde{1}, x)\|_{\alpha, 2}^{2}=\sum_{m=0}^{\infty}(1+m)^{\alpha} A_{m}(x)
$$

- The terms $A_{m}(x)$ with $m=1, \ldots,\left[\frac{\lambda H}{G}\right]$ are controlled by

$$
c \gamma^{-\lambda}\left\{g_{N-1}(\gamma, \lambda)+g_{N-1}\left(\gamma, \frac{\lambda}{2}+\frac{\epsilon G}{2 H}-\frac{1}{M}\right)\right\} m^{d\left(1-\frac{8 \beta-1}{6}\right)-1}
$$

where $\gamma=\left(\frac{1}{2}-\beta\right)|x|^{2}$, and $\varepsilon$ and $M$ satisfy

$$
\frac{\lambda}{2}+\frac{\epsilon G}{2 H}-\frac{1}{M}>0
$$

Then, by Lemma 6, part 1, this behaves asymptotically, when $\gamma \downarrow 0$, as $c \gamma^{-\lambda}\left(\log \frac{1}{\gamma}\right)^{N-1}$ if $\lambda>0$, and as $c\left(\log \left(\frac{1}{\gamma}\right)\right)^{N}$ if $\lambda=0$. 
- The terms $A_{m}(x)$ with $m \geq\left[\frac{\lambda H}{G}\right]+1$ are controlled by

$$
c m^{d\left(1-\frac{8 \beta-1}{6}\right)-1} \gamma^{-\lambda} g_{N-1}(\gamma, \lambda) m^{-\frac{N}{2 H}} .
$$

Then

$$
\begin{aligned}
\sum_{m>\frac{\lambda H}{d}}(1+m)^{\alpha} A_{m}(x) \leq c[ & \left.\sum_{m>\frac{\lambda H}{d}} m^{d\left(1-\frac{8 \beta-1}{6}\right)-1} m^{-\frac{N}{2 H}}(1+m)^{\alpha}\right] \\
& \times \gamma^{-\lambda} g_{N-1}(\gamma, \lambda),
\end{aligned}
$$

and using the fact that $\alpha<\frac{N}{2 H}-\frac{d}{2}$, we have that the series between keys is convergent and the asymptotic behavior of the last expression is, by Lemma 6 , as $c \gamma^{-\lambda}\left(\log \frac{1}{\gamma}\right)^{N-1}$ if $\lambda>0$, and $c\left(\log \frac{1}{\gamma}\right)^{N}$ if $\lambda=0$. Finally,

$$
\begin{aligned}
A_{0}(x) & =\frac{1}{(2 \pi)^{d}} B_{0}\left(\frac{1}{2}|x|^{2}\right) \\
& =\frac{1}{(2 \pi)^{d}} \frac{1}{((N-1) !)^{2}} \frac{1}{(2 H)^{2 N}} \gamma^{-\lambda} g_{N-1}\left(\gamma, \frac{\lambda}{2}\right)^{2},
\end{aligned}
$$

where $\gamma=\frac{|x|^{2}}{2}$.

Summarizing, if $N \geq 2$ and $\lambda>0, A_{0}(x)$ goes to $\infty$ when $\gamma \downarrow 0$, as $c \gamma^{-\lambda}\left(\log \frac{1}{\gamma}\right)^{2 N-2}$, and this first term dominates the asymptotical behavior because all the rest goes to $\infty$ more slowly than $\gamma^{-\lambda}\left(\log \frac{1}{\gamma}\right)^{(N-1)}$. If $N=1$ and $\lambda>0$, as the logarithm disappears, the proof only says that $\|L(1, x)\|_{\alpha, 2}\left(|x|^{-\lambda}\right)^{-1}$ is bounded by a positive constant.

If $\lambda=0, A_{0}(x)$ goes to $\infty$ when $\gamma \downarrow 0$, as $c\left(\log \frac{1}{\gamma}\right)^{2 N}$, and the rest as $c\left(\log \frac{1}{\gamma}\right)^{N}$, so also the first term dominates the asymptotical behavior. Note that we consider $A_{0}^{\frac{1}{2}}$ in place of $A_{0}$ to get the functions that appear in the theorem.

The $\lambda<0$ case follows directly. As we have seen before,

$$
\sum_{m \geq 1}(1+m)^{\alpha} A_{m}(x)
$$

is controlled by $\gamma^{-\lambda} g_{N-1}(\gamma, \lambda)$, and by Lemma 6 , part 3 , this term goes to a constant when $\gamma \downarrow 0$. 
In this case the norm $\|L(t, x)\|_{\alpha, 2}$ is continuous. Therefore we don't have an explosion, and

$$
\lim _{|x| \rightarrow 0}\|L(\widetilde{1}, x)\|_{\alpha, 2}=\|L(\widetilde{1}, 0)\|_{\alpha, 2}=\left(\sum_{m=0}^{\infty}(1+m)^{\alpha} A_{m}(0)\right)^{\frac{1}{2}}
$$

where

$$
A_{m}(0)=\frac{1}{(2 \pi)^{d}}\left(\sum_{n_{1}+\cdots+n_{d}=m} \prod_{i=1}^{d} n_{i} ! \mathbf{H}_{n_{i}}(0)^{2}\right) B_{m}(0),
$$

and

$$
\begin{aligned}
B_{m}(0) & =2^{N} \int_{[0,1]^{N}}\left(\int_{[0,1]^{N}} \underline{u}^{1-2 H d} d u_{1} \cdots d u_{N}\right) \prod_{j=1}^{N} \frac{R_{H}\left(1, z_{j}\right)^{m}}{z_{j}{ }^{H(m+d)}} d z_{1} \cdots d z_{N} \\
& =2^{N}\left(\int_{0}^{1} u^{1-2 H d} d u\right)^{N}\left(\int_{0}^{1} Q_{H}(z)^{m} \frac{d z}{z^{d H}}\right)^{N} \\
& =\left(\frac{1}{1-H d}\right)^{N}\left(\int_{0}^{1} Q_{H}(z)^{m} \frac{d z}{z^{d H}}\right)^{N} .
\end{aligned}
$$

Note that as $\lambda<0,1-2 H d>-1$.

Finally,

$$
\begin{aligned}
\|L(\widetilde{1}, 0)\|_{\alpha, 2}^{2}= & \frac{1}{(2 \pi)^{d}}\left(\frac{1}{1-H d}\right)^{N} \sum_{m=0}^{\infty}(1+m)^{\alpha} \\
& \times\left(\sum_{n_{1}+\cdots+n_{d}=m} \prod_{i=1}^{d} n_{i} ! \cdot \mathbf{H}_{n_{i}}(0)^{2}\right)\left(\int_{0}^{1} Q_{H}(z)^{m} \frac{d z}{z^{d H}}\right)^{N} \\
= & \frac{1}{(2 \pi)^{d}}\left(\frac{1}{1-H d}\right)^{N} \sum_{r=0}^{\infty}(1+2 r)^{\alpha} \\
& \quad \times\left(\sum_{r_{1}+\cdots+r_{d}=r} \prod_{i=1}^{d} \frac{\left(2 r_{i}\right) !}{\left(r_{i} !\right)^{2} 2^{2 r_{i}}}\right)\left(\int_{0}^{1} Q_{H}(z)^{m} \frac{d z}{z^{d H}}\right)^{N},
\end{aligned}
$$

because $\mathbf{H}_{2 n}(0)=\frac{1}{2^{n} n !}$ and $\mathbf{H}_{2 n+1}(0)=0$.

By the continuity of the norm, it is not necessary to prove the convergence of this series.

Remark 9. Xiao and Zhang [7] proved that when $H d<1$, that is $\lambda<0$, $B^{H}$ has a jointly continuous local time. 


\section{$\S 5$. Renormalization of the local time when the time tends to infinity}

We can also deduce the behavior of the local time $L(t, x)$ when $\underline{t}=$ $t_{1} \cdots t_{N} \rightarrow \infty$ and $|x| \rightarrow 0$. We also have to distinguish the three cases, namely $\lambda>0, \lambda=0$ and $\lambda<0$.

The precise result is the following:

Theorem 10. Let $\left\{L(t, x): t \in[0, \infty)^{N}, x \in \mathbb{R}^{d} \backslash\{0\}\right\}$ be the local time of the $(N, d)-f B m B^{H}$. Let $\lambda=d-\frac{1}{H}$. Then the following limits hold for any $\alpha<\frac{N}{2 H}-\frac{d}{2}$ :

1) For $\lambda>0$ and $N \geq 2$,

$$
\lim _{\underline{t} \rightarrow \infty,|x| \rightarrow 0}\|L(t, x)\|_{\alpha, 2}\left(\frac{2^{\frac{\lambda}{2}}\left(\frac{1}{2 H}\right)^{N}|x|^{-\lambda}}{(2 \pi)^{\frac{d}{2}}(N-1) !}\left(\log \frac{2 \underline{t}^{2 H}}{|x|^{2}}\right)^{N-1} \Gamma\left(\frac{\lambda}{2}\right)\right)^{-1}=1 .
$$

If $N=1,\|L(t, x)\|_{\alpha, 2}$ explodes when $x$ tends to 0 and $t$ to $\infty$, and independently of $t \in \mathbb{R}_{+},\|L(t, x)\|_{\alpha, 2} \cdot\left(|x|^{-\lambda}\right)^{-1}$ is bounded by a positive constant.

2) For $\lambda=0$,

$$
\lim _{\underline{t} \rightarrow \infty,|x| \rightarrow 0}\|L(t, x)\|_{\alpha, 2}\left(\frac{\left(\frac{1}{2 H}\right)^{N}}{(2 \pi)^{\frac{d}{2}} N !}\left(\log \frac{2 \underline{t}^{2 H}}{|x|^{2}}\right)^{N}\right)^{-1}=1 .
$$

3) For $\lambda<0$,

$$
\lim _{\underline{t} \rightarrow \infty,|x| \rightarrow 0}\|L(t, x)\|_{\alpha, 2}\left(\underline{t}^{(1-d H)}\|L(\widetilde{1}, 0)\|_{\alpha, 2}\right)^{-1}=1 .
$$

Proof. From the Wiener chaos expansion of $L(t, x)$ proved in [1], we can obtain that

$$
\|L(t, x)\|_{\alpha, 2}^{2}=\underline{t}^{2(1-d H)}\left\|L\left(\widetilde{1}, \underline{t}^{-H} x\right)\right\|_{\alpha, 2}^{2},
$$

and the conclusion follows from the results of the previous section.

Acknowledgment. The authors wish to express their thanks to the referee for all his helpful suggestions and comments. Also M. Eddahbi is grateful to the Centre de Recerca Matemàtica (IEC) and the Department of Mathematics of the UAB, Bellaterra, Barcelona, Spain for the kind support and hospitality he received while this work was carried out. 


\section{REFERENCES}

[1] M. Eddahbi, R. Lacayo, J. L. Solé, C. A. Tudor and J. Vives, Regularity of the local time for the d-dimensional fractional Brownian motion with $N$-parameters, to appear in Stoc. Analysis and Appl., (2005).

[2] Y. Hu and B. Oksendal, Chaos expansion of local time of fractional brownian motion, Stochastic Analysis and Applications, 20 (2002), no. 6, 815-837.

[3] P. Imkeller and F. Weisz, The asymptotic behaviour of local time and occupation integrals of the $N$-parameter Wiener process in $\mathbb{R}^{d}$, Prob. Theo. Rela. Fields, 98 (1994), 47-75.

[4] P. Imkeller, V. Perez-Abreu and J. Vives, Chaos expansion of double intersection local time of Brownian motion in $\mathbb{R}^{d}$ and renormalization, Stoc. Proc. Appl., 56 (1995), no. $1,1-34$.

[5] D. Nualart, Stochastic calculus with respect to fractional brownian motion and applications, Contemporary Mathematics, 336 (2003), 3-39.

[6] S. Watanabe, Lectures on Stochastic Differential Equations and Malliavin calculus, Springer, 1984.

[7] Y. Xiao and T. Zhang, Local times of fractional brownian sheets, Prob. Theo. Rela. Fields, 124 (2002), 204-226.

\section{Eddahbi}

Département de Mathématiques et Informatique

Faculté des Sciences et Techniques, Université

Cadi Ayyad, B. P. 549 Marrakech

Maroc

R. Lacayo

Departament de Matemàtiques

Universitat Autònoma de Barcelona

08193 Bellaterra, Barcelona

Spain

J. L. Solé

Departament de Matemàtiques

Universitat Autònoma de Barcelona

08193 Bellaterra, Barcelona

Spain

J. Vives

Departament de Matemàtiques

Universitat Autònoma de Barcelona

08193 Bellaterra, Barcelona

Spain 
C. A. Tudor

Samos/Matisse. 90, rue de Tolbiac

Université de Panthéon-Sorbonne. Paris 1

75634 Paris Cedex 13

France 